Matthews, P. B. C. \& Rushworth, G. (I957b). F. Physiol. 135, 263.

Matthews, P. B. C. \& Rushworth, G. (1958). F. Physiol. 140, 42 I.

Pollock, L. J. \& Davis, L. E. (I923). Arch. Neurol. Psychiat. I0, 39 I.

Pollock, L. J. \& Davis, L. E. (I930). F. comp. Neurol. 50, 377.

Rushworth, G. (1960). F. Neurol. Neurosurg. Psychiat. 23, 99.

Rushworth, G. (I96I). Int. F. Neurol. 2, 34.

Rushworth, G. \& Young, R. R. (1966). ' f. Physiol. (in the press).

SHERRINGTON, C. S. (1893). Philos. Trans. 184B, 631.

SHERrington, C. S. (1898). F. Physiol. 22, 319.

WALSHE, F. M. R. (1924). Brain, 47, I59.

\title{
FLEXION REFLEX AND STRETCH REFLEX IN SPASTICITY
}

\author{
(A Trial with a Polysynaptic Inhibitor) \\ By G. M. Pool, M.D. \\ Zuiderziekenhuis, Rotterdam
}

Introduction. On the basis of the functional organisation of the spinal cord, there are two reflex patterns (Harpuder, 1962):

I. the stretch reflex, subserving posture, and maintenance of posture in locomotion;

2. the general flexion reflex, subserving withdrawal and protection against injury.

The stretch reflex (fig. I) originates in the annulo-spiral ring of the muscle spindles which lie in parallel with the main muscle fibres and participate in their attachments. The spindle afferent connects by single synapse with the motoneuron innervating the main muscle fibres. The spindle afferents are inhibited by active muscle contraction, activated by passive stretch. By way of the socalled spindle loop, the muscle shortens reflexly until it has shortened to the same extent as has the spindle. The spindle discharge depends not only upon stretch but also upon the innervation which regulates the sensitivity of the spindles. This innervation is for the most part by small fibres of the so-called gamma system and controlled by the reticular formation and further on by higher centres.

Of the polysynaptic reflexes, the polysynaptic flexion reflex plays an important role in the walking action, having its receptive field on the skin of the sole of the foot. Stimulation of sufficient strength of this area causes a co-ordinated withdrawal action with flexion in knee and hip, and extension of the contralateral leg.

In the walking action there is an exact interplay

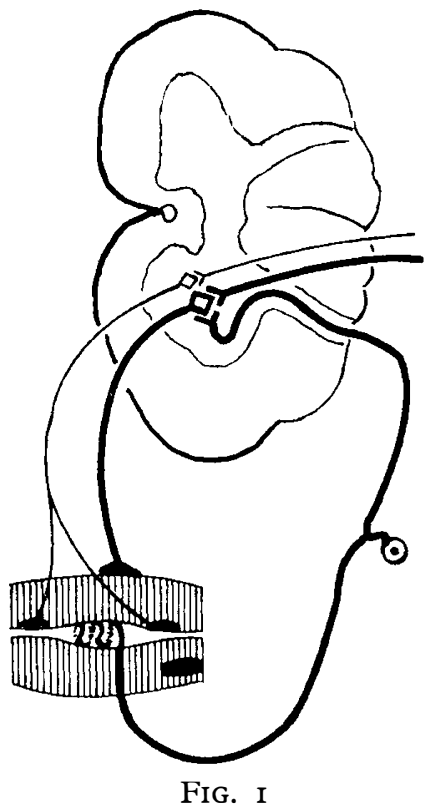


between polysynaptic movement reflexes and monosynaptic posture reflexes. The first having their receptive field on the skin, the second in the sensory receptor of the muscle spindle.

In case of a paraplegia, the flexion reflex can have a widened receptive field, even on to the anterior abdominal wall (Marshall, I954). If the spinal cord is gravely injured but does not suffer complete division-some of the descending fibres, especially those of the vestibulo- and reticulo-spinal tracts, may have escaped injury and so the control of the supra-segmental centres on the spindles may have remained intact. The answer to the more easily evoked flexion reflex because of the widened receptive field in order to maintain posture in that case should be an increase in tone. We usually call that a spastic condition. There are many conditions in which the interplay between the reflexes is disturbed and in which the increase in tone is so high that it interferes with our object to get the patient back to daily life.

In the fight against these conditions we know different orthopaedic and neurosurgical operations; the pharmaceutical industries show their interests too. They brought out drugs working more centrally as for example chlorpromazine, and drugs which should have an inhibiting effect on the polysynaptic reflexes. Many tests have been invented to control these drugs, but it is difficult to come to an objective measurement, which is very necessary.

Pedersen and Schleisner (1959) published good results of a drug that would inhibit the polysynaptic reflexes and the gamma afferents (stretch reflex) and which gave an improvement of bladder control. The drug was a CIBA preparation: 2-hydrazino-4,6-bis-diethylamino-I,3,5-triazine dihydrochloride. No serious sideeffects were observed. They tested the drug in 50 patients, mainly with spastic paraparesis, and found in half of the cases an appreciable relaxation of muscle tone, an improvement in the gait, and an excellent effect on the disturbances of bladder control. They controlled the effect of the drug on the patient's gait which, indeed, could be a measurement of the effect on the interplay between polysynaptic movement reflexes and the monosynaptic posture reflexes. They did not test the drug in a double-blind, as they did not expect a reverse of symptoms from a temporary discontinuance.

Clinical Trial and Methods. This drug we employed in six children with a so-called cerebral palsy, and although there seemed to be an improvement of the gait, of some of them, this effect was also obtained without administration of the drug and probably this was due to a training effect (figs. 2 and 3 ).

The time needed for 70 metres in stretches of IO, and the best performances of a Io-metre stretch were weekly recorded. Seventy metres being the distance they all could endure without difficulty. A similar drug, which on animals had an even stronger effect, was tested in a double-blind test. By way of an electrical stimulus the flexion reflex of the leg was recorded electromyographically in $2 \mathrm{I}$ patients with different spinal lesions:

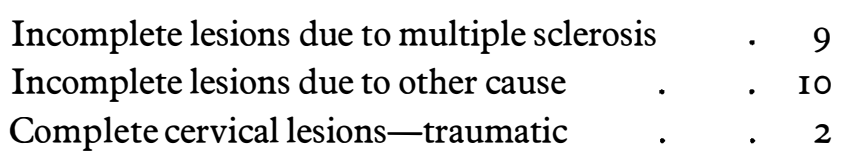




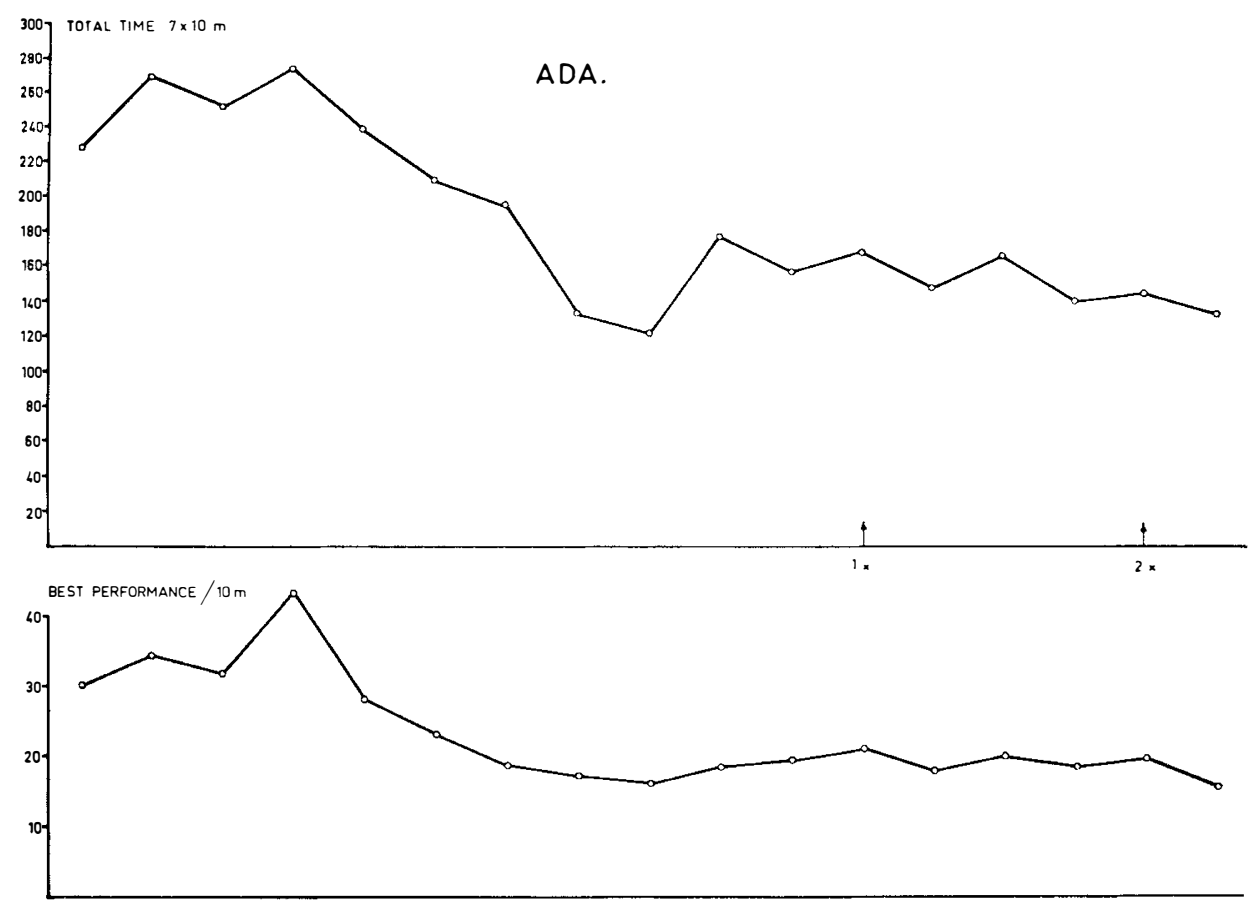

FIG. 2

Total time and best performance in seconds daily control. Arrows indicate daily dose $x$ $50 \mathrm{mg}$.

FIG. 3

Total time and best performance in seconds weekly control. Arrows indicate daily dose $\times 50 \mathrm{mg}$.
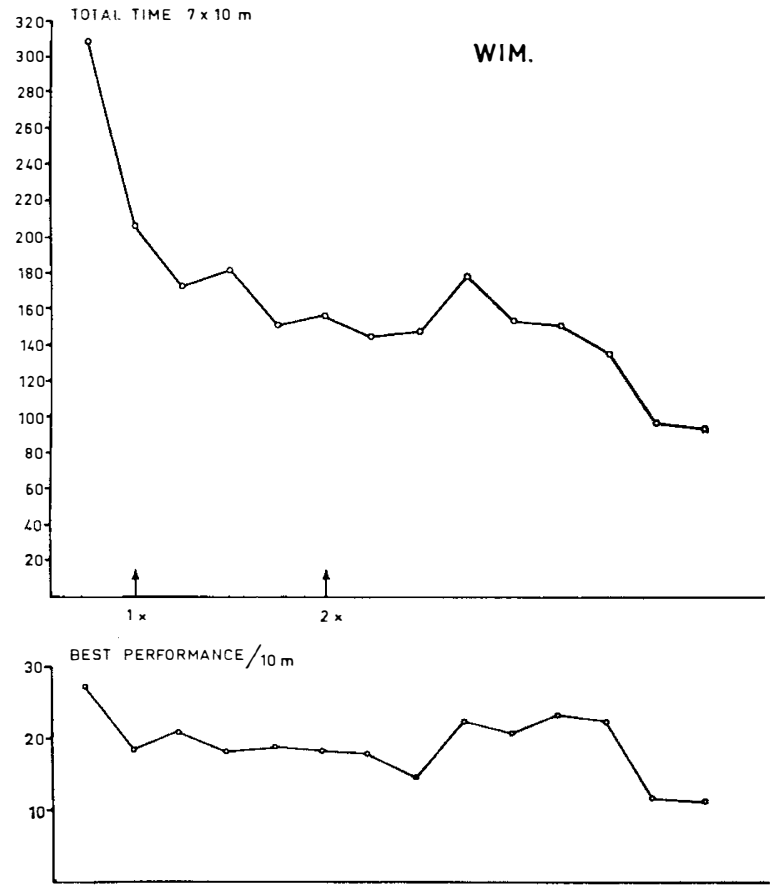
Two needles were inserted under the skin of the sole of the foot without an anaesthetic; electrical stimuli were applied through a Disa stimulator. The strength of

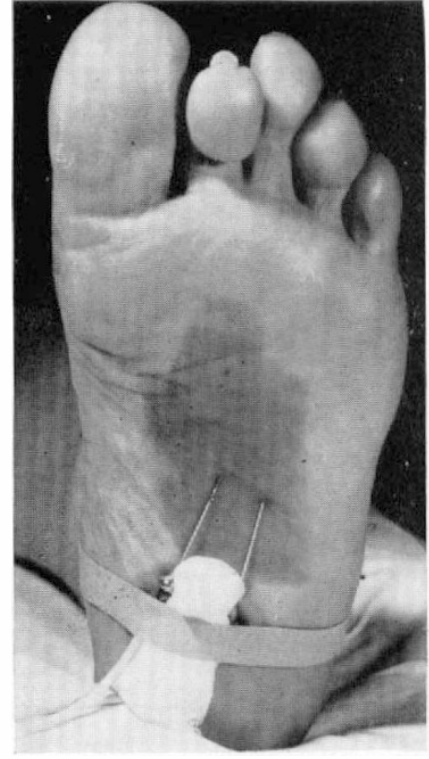

FIG. 4 the square wave pulse was kept constant in different examinations.

The reflex responses were recovered bipolar from the $\mathrm{m}$. rectus femoris, which is involved in the flexion action serving as a flexor of the hip (Kugelberg et al., 1960).

Of the patients, Io complained of drowsiness, 4 of them, therefore, discontinued the medication, 7 of the remainder had a subjective improvement.

Although a myographic response was probable in some of them, we could not come to an objective measurement, because of the difference in response obtained on different heights above the patella in the $\mathrm{m}$. rectus femoris and because of the difference in the clinical picture of our patients.

Three myographic patterns were obtained (Pool, 1965), but also remarkable was that:

I. In all of our patients and in a control person there was a withdrawal action on insertion of the needles under the skin of the sole of the foot;

2. Only in two of our patients electromyographic activity remained in the $\mathrm{m}$. rectus femor is after the insertion of the needles under the skin of the sole of the foot;

3. In all of our patients there was electromyographic activity after an electrical stimulus of sufficient strength was applied;

4. The electromyographic pattern obtained in 17 patients did not seem to differ grossly from that of a control person;

5. In two patients, having a total cervical lesion, two bursts of action potentials were obtained, separated by a rest period: the first due to the flexion action, the second only appeared when the leg was allowed to return freely to the rest position, and is probably due to the stretch reflex.

So electromyographic reaction was only obtained in all our patients on insertion of the needles under the skin of the sole of the foot and on applying the electrical stimulus.

\section{DISCUSSION}

Kugelberg (1948) made an electromyographic study of the flexion reflex with different stimuli and observed that the reflex consists of several parts according to the different conduction speeds in afferent $\mathrm{A}$ and $\mathrm{C}$ fibres. He controlled the reflex with stimuli which have a moving character, as stroking with a needle, with heat and with an electrical stimulus. From the work of Kugelberg it can be 
concluded that the flexion reflex has different kinds of stimuli. Kugelberg also states that the first sign of weakened control from supraspinal levels is the A fibre flexion reflex, the slow response being more easily inhibited.

Marshall (1954) in his studies of flexion and extension reflexes also found a difference of reflexes obtained, according to the way he applied the stimulus. He dragged a bodkin across the skin and found a difference in reaction between a short drag with heavy pressure and a prolonged drag with moderate pressure, which is a more moving type of the stimulus. Marshall also noticed that the noxious aspect of the stimulus was not indispensable: in some of his patients with well-developed flexion reflexes, blowing on the sole of the foot was sufficient to produce the reflex. This blowing stimulus can be looked upon as having a moving character. In the literature there is an agreement that patients with a paraplegia have a widened receptive field (Marshall, I954; Kugelberg et al., 1960; Harpuder, 1962). It is also very often stated that the reflex is a protective withdrawal reflex and serves to defend against injury. It is, however, not clear in patients with a paraplegia, lying in their bed and having spontaneous flexion contractions of their legs, against what injury they are trying to protect themselves. On the contrary, sometimes they even injure their legs by this action! The needles in the sole of the foot of our patients did evoke the reflex only while being inserted.

In a patient with a cervical lesion $\mathrm{C}_{5}-\mathrm{C} 6$ we found that about the same strength of electrical stimulus with needles inserted under the skin of the sole of the foot was needed as in a control person. In the paraplegic patient, however, slight stroking and even blowing along the skin elicited the reflex too. He 'protected' his legs both against a slight stimulus with a moving character along a much widened field and against a stimulus of noxious character for which the same strength as in a control person was needed.

Because of the widened field, there is a much greater possibility of a moving character of the normally present stimuli and because of the diminished control, the flexion reflex is more easily evoked. To maintain posture, it can be expected that the stretch reflex reacts on the withdrawal action with an increase in tone, which counteracts the withdrawal movement. The combination of the two reflexes, withdrawal in flexion and increase in tone, produces the so-called spastic contractures.

Besides the interplay of flexion and stretch reflex, there is probably the intervention of stimuli from visera, bladder, tendons, etc., and the stimuli coming from the contralateral leg. In patients with a total cervical lesion, the interplay between flexion and stretch reflex is totally disturbed. The action of the stretch reflex is postponed until sufficient passive stretch in the muscle is reached.

In these patients, the stretch reflex is only of spinal origin, no central tone control being left. More pronounced flexion contractures can be expected with them.

According to Rushworth (1964), Sherrington has taught that nothing comes out of the central nervous system unless some information is put into it, and Sherrington also stressed the importance of analysing the stimulus when investigating motor phenomena. In case of a weakened control and a widened receptive field, reducing the normally present stimuli, especially those with a moving character from which the leg tends to withdraw in flexion, would serve as a polysynaptic inhibitor of the flexion reflex of the lower limb. 


\title{
SUMMARY
}

The interplay between stretch reflex and flexion reflex is discussed. A drug, which should have an inhibiting effect on the polysynaptic flexion reflex and on the gamma afferents was examined in walking tests in six children with cerebral palsy. The improvement obtained was probably due to a training effect. In $2 \mathrm{I}$ patients with different spinal cord lesions the flexion reflex of the lower limb was examined electromyographically by way of an electric nociceptive stimulus with needles inserted under the skin of the sole of the foot. This was done in a doubleblind test, but no objective measurement could be obtained.

The flexion reflex was only obtained when the stimulus had a moving or nociceptive character. It was concluded that the flexion reflex probably has more qualities than withdrawal in defence. The importance of the widened receptive field in patients with paraplegia and a moving character of stimulus are stressed.

\section{ACKNOWLEDGMENTS}

I am indebted to Dr. M. de Vlieger and to many members of his department for their help with the electromyographic examinations. The tablets and dummies were kindly provided by CIBA Ltd.

\section{REFERENCES}

HARPUDER, K. (Oct. 1962), Proceedings of Clinical Spinal Cord Injury, Veterans Administration, p. 32.

KugelberG, E. (1948). Brain, 7I, 304.

Kugelberg, E., EKLund, K. \& Grimsby, L. (1960). Brain, 83, 394.

MARShall, J. (1954). Brain, 77, 290.

Pedersen, E. \& Schleisner, P. (I959). Acta psychiat. scand. 34, 342.

Pool, G. M. (1965). Int. f. Paraplegia, 3, I88.

Rushworth, G. (1964). Ann. phys. Med. Suppl.

\section{SPASTICITY IN THE UPPER LIMB}

\author{
J. R. SILVER, M.B., B.S., M.R.C.P.E. \\ Liverpool Regional Paraplegic Centre, Southport
}

ONE of the results of the increase of wealth of society is that many more people are now owning and using motor-cycles and cars. This has resulted in an increased number of road accidents, some of these naturally involve the cervical spine and cause tetraplegia. The better understanding of the treatment of these injuries during the last 20 years, particularly with reference to the management of the chest and urinary tracts, means that many more tetraplegics are surviving. The long-term prognosis depends largely upon preventing chest and renal infections and pressure sores. The useful contribution that they can make to society and to eading a fuller and freer life depends almost entirely upon the useful function 